\title{
Automatic Detection of Arrhythmias Using a YOLO Based Network with Long-Duration ECG Signals ${ }^{\dagger}$
}

\author{
Won Hee Hwang 1,2, Chan Hee Jeong ${ }^{1}$, Dong Hyun Hwang ${ }^{1}$ and Young Chang Jo 1,* \\ 1 Korea Electronics Technology Institute, Korea; anne0615@keti.re.kr (W.H.H.); chjeong@keti.re.kr (C.H.J.); \\ dhhwang89@keti.re.kr (D.H.H.) \\ 2 School of Electrical and Electronic Engineering, Yonsei University, Seoul, Korea \\ * Correspondence: ycjo@keti.re.kr \\ + Presented at the 7th Electronic Conference on Sensors and Applications, 15-30 November 2020; Available \\ online: https://ecsa-7.sciforum.net/.
}

Published: 15 November 2020

\begin{abstract}
Early detection of arrhythmia is very important. Recently, a wearable device is used to monitor the patient's heartbeat to detect arrhythmia. However, there are not satisfied algorithms for real-time monitoring arrhythmia in a wearable device. In this work, A novel Fast and Simple Arrhythmia detection algorithm based on YOLO is proposed. The algorithm can detect each heartbeat on long duration ECG signals without R-peak detection and can classify arrhythmia simultaneously. The model replaces the 2D CNN with 1D CNN and a bounding box with a bounding window to utilize Raw ECG signal. Results demonstrate that the proposed algorithm has high performance on speed and mAP in detecting Arrhythmia. Furthermore, the bounding window can predict different window lengths on different types of arrhythmia. Therefore, the model can choose optimal heartbeat window length for Arrhythmia classification. Since the proposed model is a compact 1D CNN model based on YOLO, it can be used in a wearable device and embedded system.
\end{abstract}

Keywords: arrhythmia; electrocardiogram; YOLO; deep learning; real time; wearable device

\section{Introduction}

Arrhythmia refers to a group of conditions that cause the heart to beat rapid or irregular. It is detected by Electrocardiogram which measures the state of the heart's electrical activity. In general, the process of Arrhythmia detection is performed in three steps: Beat segmentation, feature extraction and classification. Conventional Heartbeat segmentation is the process of $\mathrm{R}$ peak detection in a long duration of ECG. Differentiation [1], Digital filter [2] is used in R peak detection. The R peak detection algorithm is especially emphasized in the bio signal wearable devices for computational cost [3].

Feature extraction is the following step in beat segmentation. In machine learning, Wavelet transform (WT) [4], Independent component analysis (ICA) [5] are used. Feature extraction reduces the dimensions of beat feature and can greatly improve classification performance. Classification is the process of classifying the beat to each class based on the feature. However, most of the algorithms are handcrafted features and such a pipeline processing method involves a problem of loading data into memory in each step and a problem of real-time execution due to operation processing.

Deep learning based method overcame this problem by end to end learning which feature extraction and classification are fused into one model. Jun [6] used 2D CNN Model which transform the ECG beat into ECG. Ji [7] also proposed Faster R-CNN which converts the ECG signal into an ECG image. They have a 99.21\% average accuracy. Kiranyaz [8] used 1D CNN for classification of 5 types of arrhythmia. They made real time classification model using 3 consecutive 1D CNN layers. But deep learning based method for classifying arrhythmia still need beat segmentation process. 
In this paper, we proposed 1D YOLO model which can detect arrhythmia without $\mathrm{R}$ peak in long ECG sequence. The model can detect more than one type of arrhythmia in long sequence. We modified Yolo model into 1D Yolo and bounding box is replaced with bounding window. As a result, the model predicts only one coordinates for bounding window.

\section{Related Work}

YOLO

YOLO is an effective real-time object detection Deep learning model [10]. The model applies a single neural network to the input image and divides the image into S X S Grid cell. Grid cell approach is faster than Selective Search and Edge Boxes in region proposal. Fast RCNN use Selective search and Faster RCNN adapts Edge boxes for region proposal but these kinds of region proposal is not enough to use in real time system. Therefore, YOLO is widely used in real time object detection.

\section{Materials and Methods}

\subsection{MIT-BIH Database}

In this study, MIT-BIH arrhythmia database [9] is used for training and testing. The heart beat are classified into 5 types: Normal, right bundle branch block (RBBB), left bundle branch block (LBBB), ventricular ectopic beat (VEB), supraventricular ectopic beat (SVEB). Since this is imbalanced, we downsample the normal type to match the overall number of other types of arrhythmia.

MIT-BIH arrhythmia database contains 48 records and each record is about $30 \mathrm{~min}$. We split 30 min ECG data into $10 \mathrm{~s}$ ECG segments. We do not use the segment which has only normal type. Therefore, each segment has more than one type of arrhythmia.

\subsection{Proposed 1D YOLO Model}

The overview of proposed 1D YOLO is shown in Figure 1. We divide 36 grids in ECG segment and each grid predicts 2 bounding windows. Therefore, 72 bounding window is predicted in each 10s ECG segment with confidence score and position. Most of bounding windows has low confidence score and it means bounding window detect background. We wanted to focus on the bounding window which has ECG beat. To remove the bounding window which has low score, the bounding window is sorted according to confidence scores from large to small. We set a threshold and the confidence score under this threshold is deleted.

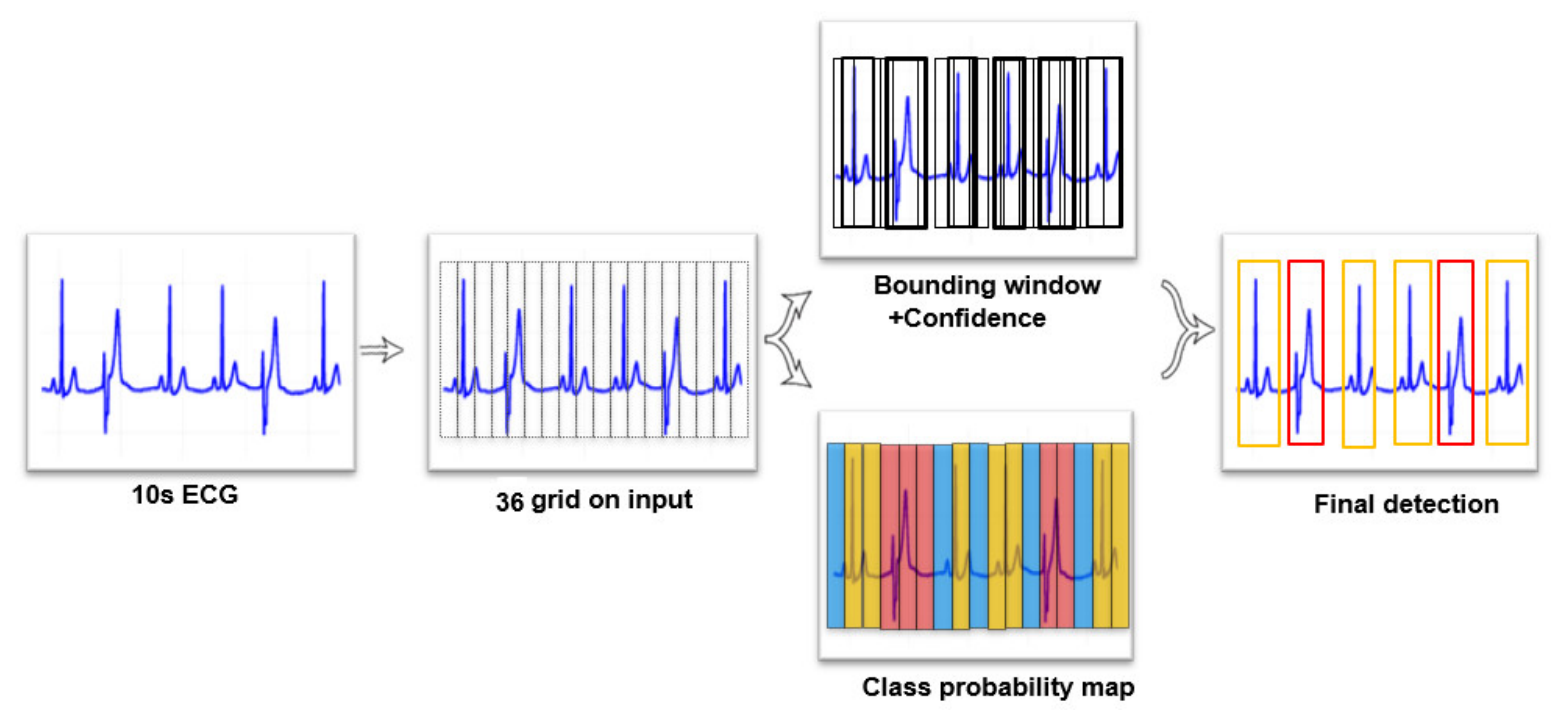

Figure 1. Proposed YOLO model detection. 
The post-processing is needed to remove several detections on the same heartbeat. Non Maximum Suppression is used to remove the multiple bounding windows on the same heartbeat and the bounding window which has a high confidence score has left. The results are shown in Figure 2.

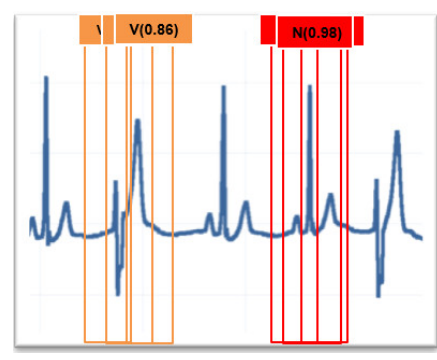

(a)

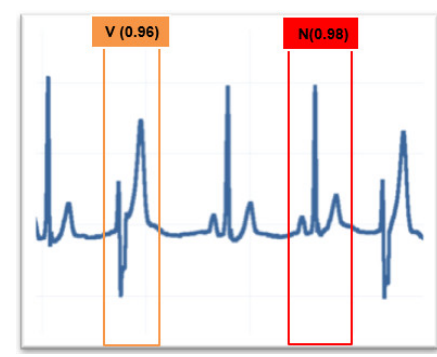

(b)

Figure 2. (a) Before Non Maximum Suppression; (b) After Non Maximum Suppression;.

\section{Result and Discussion}

The proposed YOLO model was examined using MIT-BIH database. We divide 5 types of Arrhythmia. Precision and recall of each arrhythmia is shown Table 1. The proposed model is able to correctly detect 5 types of arrhythmia at average precision of 0.97 , average recall of 0.95 and $0.96 \mathrm{~F} 1$ score. However, SVEB has low recall value. This may be due to the less dataset of SVEB compared to other types of arrhythmia. In addition, SVEB is characterized not only morphological features, but also beat-to-beat feature. This made the model difficult to detect SVEB. The entire detection time of proposed 1D YOLO on $10 \mathrm{~s}$ ECG segment is $0.03 \mathrm{~s}$. This is real time realizable.

Table 1. Number of beats in each arrhythmia type.

\begin{tabular}{cc}
\hline Type & No. Beats \\
\hline Normal & 25,891 \\
LBBB & 8021 \\
RBBB & 7160 \\
SVEB & 2753 \\
VEB & 6947 \\
\hline
\end{tabular}

Table 2. Precision and Recall of each arrhythmia types.

\begin{tabular}{ccc}
\hline Type & Precision & Recall \\
\hline Normal & 0.97 & 0.98 \\
LBBB & 0.99 & $0.98^{1}$ \\
RBBB & 0.99 & 0.99 \\
SVEB & 0.96 & 0.86 \\
VEB & 0.97 & 0.96 \\
\hline
\end{tabular}

Table 3. Overall performance of proposed model.

\begin{tabular}{ccccc}
\hline & Precision & Recall & F1 Score & mAP \\
\hline 1D YOLO & 0.97 & 0.95 & 0.96 & 0.96 \\
\hline
\end{tabular}

\section{Conclusions}

In this study, we proposed a YOLO-based arrhythmia classification model that can detect each heartbeat and classify it as arrhythmia on 10 s ECG segment without a beat extraction step. By processing the bit extraction step, feature extraction, and classification step as a single model, the complexity of computation was reduced and presented as a model usable for embedded. We only classify 5 types of arrhythmia, but the model can be used to detect various arrhythmias in the future. 
Funding: This research received no external funding.

Acknowledgments: This work was supported by the Korean Ministry of Science \& ICT and IITP under project contract No. 2020-0-01970-001 [Development of Smart Patch and Multimodal Bio signal Analysis Algorithm for Self-monitoring of Infectious Respiratory Diseases] and partly supported by the Korean Ministry of Trade, Industry, Energy/KEIT under project contract No. 20009959 [ Development of stress care device and service technology using tES-based multi-sensory stimulation with clinical effectiveness]

Conflicts of Interest: The authors declare no conflict of interest.

\section{References}

1. Afonso, V.X.; Tompkins, W.J.; Nguyen, T.Q.; Luo, S. ECG beat detection using filter banks. Ieee Trans. Biomed. Eng. 1999, 46, 192-202.

2. Keselbrener, L.; Keselbrener, M.; Akselrod, S. Nonlinear high pass filter for R-wave detection in ECG signal. Med. Eng. Phys. 1997, 19, 481-484.

3. Arefin, M.R.; Tavakolian, K.; Fazel-Rezai, R. QRS complex detection in ECG signal for wearable devices. In Proceedings of the 2015 37th Annual International Conference of the IEEE Engineering in Medicine and Biology Society (EMBC), City, Country, Day Month 2015; pp. 5940-5943.

4. Mahmoodabadi, S.Z.; Ahmadian, A.; Abolhasani, M.D. ECG feature extraction using Daubechies wavelets. In Proceedings of the Fifth IASTED International conference on Visualization, Imaging and Image Processing, City, Country, Day Month 2005; pp. 343-348.

5. Jiang, X.; Zhang, L.; Zhao, Q.; Albayrak, S. ECG arrhythmias recognition system based on independent component analysis feature extraction. In Proceedings of the TENCON 2006-2006 IEEE Region 10 Conference, City, Country, Day Month 2006; pp. 1-4.

6. Jun, T.J.; Nguyen, H.M.; Kang, D.; Kim, D.; Kim, D.; Kim, Y.H. ECG arrhythmia classification using a 2-D convolutional neural network. Arxiv Prepr. 2018, arXiv:1804.06812.

7. Ji, Y.; Zhang, S.; Xiao, W. Electrocardiogram classification based on faster regions with convolutional neural network. Sensors 2019, 19, 2558.

8. Kiranyaz, S.; Ince, T.; Gabbouj, M. Real-time patient-specific ECG classification by 1-D convolutional neural networks. IEEE Trans. Biomed. Eng. 2015, 63, 664-675.

9. Moody, G.B.; Mark, R.G. The impact of the MIT-BIH arrhythmia database. IEEE Eng. Med. Biol. Mag. 2001, 20, 45-50.

10. Redmon, J.; Divvala, S.; Girshick, R.; Farhadi, A. You only look once: Unified, real-time object detection. In Proceedings of the IEEE Conference on Computer Vision and Pattern Recognition, City, Country, Day Month 2016.

11. Hwang, W.H.; Jeong, C.H.; Hwang, D.H.; Jo, Y.C.; Choi, Y.S.; Hong, H.K. Real time detection of Arrhythmia using YOLO base model. In Proceedings of the Conference on Information and Control System, City, Country, Day Month 2020.

Publisher's Note: MDPI stays neutral with regard to jurisdictional claims in published maps and institutional affiliations.

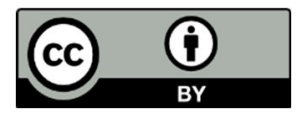

(C) 2020 by the authors. Submitted for possible open access publication under the terms and conditions of the Creative Commons Attribution (CC BY) license (http://creativecommons.org/licenses/by/4.0/). 